\title{
Raman spectroscopy on microcalcifications reveals peculiar differences between male and female breast cancer
}

Alessandro Caldarone ${ }^{1 \#}$, Francesca Piccotti ${ }^{1 \#},{\text { Carlo } \text { Morasso }^{1}, \text { Marta Truffi }^{1}, \text { Federico Sottotetti }}^{1}$, Chiara Guerra ${ }^{1}$, Sara Albasini ${ }^{1}$, Manuela Agozzino ${ }^{1}$, Laura Villani ${ }^{1}$, Fabio Corsi ${ }^{1,2} *$

1) Istituti Clinici Scientifici Maugeri IRCCS, Via Maugeri 4, Pavia, Italy

2) Department of Biomedical and Clinical Sciences "Luigi Sacco", University of Milan, Milan, Italy

\section{Corresponding author:}

Prof. Fabio Corsi, Department of Biomedical and Clinical Sciences "Luigi Sacco," University of Milan, Via G. B. Grassi, 74, 20157 Milan, Italy, Surgery Department, Breast Unit, Istituti Clinici Scientifici Maugeri Maugeri S.p.A. SB, via Maugeri 10, 27100 Pavia, Italy (e-mail: fabio.corsi@icsmaugeri.it).

\# Both authors contributed equally to this work 


\begin{abstract}
Microcalcifications (MCs) are important disease markers for breast cancer. Many studies were conducted on their characterization in female breast cancer (FBC), but no information is available on their composition in male breast cancer (MBC). Raman spectroscopy (RS) is a molecular spectroscopy technique that can explore the biochemical composition of MCs rapidly and without requiring any staining protocol. We here compared, by Raman spectroscopy, the MCs identified on breast cancer pieces from male and female patients. In this study, we used Raman spectroscopy to analyse 41 microcalcifications from 5 invasive MBC patients and 149 MCs from 14 invasive FBC patients. Our findings show that hydroxyapatite is the most abundant type of calcium both in MBC and FBC. However, some differences in the amount and distribution of calcium minerals are present between the two groups. Besides, we observed that MCs in MBC have a higher amount of organic material (collagen) than FBC.

This study provides the first overview of the composition of microcalcifications present in MBC and suggests that they have several specific features. Our result support the need for studies specifically designed to the understanding of MBC.
\end{abstract}

Keywords: Breast cancer; Male breast cancer; Microcalcifications; Hydroxyapatite; Raman spectroscopy. 


\section{Introduction}

Male breast cancer is an extremely rare type of malignancy, representing only about $1 \%$ of all breast cancer (BC) diagnoses worldwide and only about $1 \%$ of cancers occurring in men. [1,2] Despite rare, $\mathrm{MBC}$ is associated with higher mortality than female breast cancer. Men are not subjected to screening and thus MBC patients are often older than women and the disease is typically diagnosed at a later stage, with more advanced features. However, even after adjustment for clinical features and access to treatment, a higher proportion of death in males than females was reported. [3] This observation suggests that other factors may underlie such sex disparity and confirms that more studies focusing specifically on $\mathrm{MBC}$ are needed.

In fact, since the lack of recruited men in BC clinical trials, and given the relatively few studies on $\mathrm{MBC}$, treatments and diagnostic approaches for men generally derive from those for women. [4] Microcalcifications in $\mathrm{MBC}$ are rarer and poorly studied, since mammography is not routinely used on MBC patients, and a mammographic screening for males is not performed. Therefore, MCs in MBC have never been studied in detail, except for their radiological characteristics, since they usually appear rounder and coarser than in FBC. [5] But while many different studies were previously performed on women, we do not have a description of the mineral forms of calcium present in men and we do not know if there are differences in their composition that could highlight biological specificities of MBC.

Raman spectroscopy is a photonic approach that can investigate the composition of samples by irradiation with laser light. RS can provide a complete overview of the mineral constituents of MCs and it is compatible with real-time in vivo diagnostic evaluations. [6] RS was previously used in a few studies for the characterization of MCs in FBC, whereas MBC patients were never included in the studies. $[7,8,9,10]$ In this work, for the first time, we applied RS for the detailed characterization of MCs present in MBC and we compared their composition with a dataset of MCs present in FBC. 


\section{Materials and methods}

This study includes 19 patients affected by luminal invasive breast carcinoma and treated at the Breast Unit of the ICS Maugeri in Pavia, a EUSOMA-accredited, tertiary referral center involved in wide screening programs in Northern Italy, between 2013 and 2019 (5 MBC and 14 FBC). From surgical specimens of MBC patients and biopsy specimens of FBC patients, MCs were identified and analyzed on RS (see Supplementary Data for details on data acquisition and analysis). All patients had to sign an informed consent authorized by the Ethics Commission of the Institutions (Protocol 2281/2018 EC), which approved the study in accordance with the Helsinki Declaration. Only patients with a diagnosis of invasive ductal carcinoma of the breast were included. 


\section{Results}

Detailed patient characteristics are shown in Table S1-S2 in the supplementary information. 190 MCs were identified and fully scanned by RS: 41 MCs from 5 MBC patients and 149 MCs from 14 FBC [7]. By the RS analysis of MCs, some differences in the proportion of minerals and their crystal characteristics emerged.

Hydroxyapatite (HA), a particular form of calcium phosphate, appeared as the most common mineral present in both groups of microcalcifications; in particular, HA was identified in 40 male MCs (Figure 1a) and 136 female MCs (Figure 1c). Overall, HA made 92\% of the total calcium deposit found in MBC (Figure 1b) and 96\% of the one in FBC (Figure 1d). A different form of calcium phosphate named whitlockite (WIT) was detected in 2 male MCs (Figure 1a) and 6 female MCs (Figure 1c), always in association with HA. WIT resulted more abund ant in MBC $(3,1 \%)$ than in FBC $(0,35 \%)$ (Figure 1b-1d).

Calcium carbonate is present in MCs from both $\mathrm{MBC}$ and $\mathrm{FBC}$ in its crystalline form (calcite). However, while in FBC calcite was always detected as a small deposit within larger MCs made of $\mathrm{HA}$, in one MBC patient we identified a MC entirely made of calcite with a small deposit of amorphous calcium carbonate $(\mathrm{aCaCa})$ inside. At last, one male $\mathrm{MC}$ resulted made of calcium oxalate $(\mathrm{CaO})$ in combination with a smaller deposit of HA and WIT (Table 1a). This represented an anomalous result as $\mathrm{CaO}$ was not detected in our dataset of MCs from FBC (Table 1b) and, to the best of our knowledge, was never reported in the scientific literature in infiltrating FBC.

We also compared the crystal characteristics of calcium phosphate deposits identified by RS in the two groups of MCs.

The analysis of Raman spectra confirmed that MCs present MBC and FBC share a similar profile. However, the different spectrum (Figure S1) highlighted small differences in the crystal structure between the two groups. A detailed analysis revealed the significance of the spectral differences (Figure S2). In particular, the bands at $960 \mathrm{~cm}-1$, relative to the presence of phosphate groups, and the one at $1075 \mathrm{~cm}-1$, relative to carbonate, are significantly reduced in MBC than in FBC (Figure $2 \mathrm{a}-2 \mathrm{~b})$. On the contrary, the band at $1462 \mathrm{~cm}-1$ relative to the presence of organic material within MCs (mostly referring to proteins [11]), is more pronounced in MBC (Figure 2c). We also studied the ratio between the bands at $960 \mathrm{~cm}-1$ and $1073 \mathrm{~cm}-1$ that, on the contrary, did not vary between MBC and FBC (Figure 2d), suggesting that the difference in the crystal structure is driven by a different amount of proteins and other organic materials present within the MCs. 


\section{Discussion}

The role of MCs in BC is still not fully understood. However, their association with the presence of a malignant lesion is well known and MCs are an important marker of the disease. A recent study by our group proved that the mineral characteristics of MCs correlate with the histological classification of FBC making the connection between MCs and BC even tighter [7].

By using RS imaging, we now studied the different forms of calcium present in MCs from infiltrating MBC and we compared the results with the cohort of infiltrating FBC included in our previous study.

The results show that MCs in MBC resulted mainly made of $\mathrm{HA}$, as previously reported also for FBC. However, some clear differences emerged during the analysis. WIT, a different mineral form of calcium phosphate is a not negligible component in $\mathrm{MBC}$ while was present just as traces in infiltrating FBC. In one MBC, we also identified the presence of $\mathrm{CaO}$. This is particularly notable, as $\mathrm{CaO}$ is a specific marker of benign lesions and was never reported on invasive FBC. $[12,13]$ Besides, in $\mathrm{FBC} \mathrm{CaO}$ forms homogeneous calcification distinct from the one made of calcium phosphate. [14] On the contrary, here $\mathrm{CaO}$ co-localized with a deposit of HA and WIT. [15] Figure $\mathrm{S} 2$ in supporting information report the MC presenting both calcium oxalate and calcium phosphate present as HA and WIT.

The comparison of the spectral characteristics between MBC and FBC also highlighted differences in the crystal structure of MCs. MCs in MBC are richer in proteins and have less calcium. However, the ratio between the peaks of phosphate and of carbonate inclusion is unchanged between the two groups. These data suggest an active role of collagen, and thus of cancer microenvironment, on the formation of MCs and support the fact that MBC and FBC are diseases with proper characteristics that should be taken into the account. 


\section{Conclusion}

The bio-mineralization process that led to the formation of $\mathrm{MCs}$ in $\mathrm{BC}$ is currently under investigation by several groups as MCs are recognized as an early marker of BC. The present study reports the first detailed characterization of MCs present in MBC. These findings highlighted some particular features of MCs in MBC in terms of the different mineral forms of calcium and in the balance between calcium and proteins. Overall, our data suggest that different biochemical processes can influence the formation of $\mathrm{MCs}$ in $\mathrm{MBC}$ an $\mathrm{FBC}$; and support the need to design studies for the understanding of MBC. 


\section{Supplementary}

Supplementary file "ESM.pdf" contains details about the clinical cohort involved in the study and an accurate description of the experimental protocols.

\section{Declarations}

\section{Funding}

This work was not supported by any specific funding.

\section{Conflict of interest}

The authors declare that they have no conflict of interest that are relevant to the content of this article.

\section{Ethics approval}

All procedures performed in this study involving human participants were in accordance with the ethical standards of the Ethics Commission of the Institutions (Protocol 2281/2018 EC), which approved the study in accordance with the Helsinki Declaration.

This article does not contain any studies with animals performed by any of the authors.

\section{Consent to participate}

Informed consent was obtained from all individual participants included in the study.

\section{Data availability}

The data generated and analysed during the current study is available from the corresponding author on reasonable request.

\section{Authors' contribution}

C. Morasso, F. Corsi: study concepts; C. Morasso, F. Corsi, L. Villani: study design; A. Caldarone, F. Piccotti, C. Guerra, M. Agozzino: data acquisition; A. Caldarone: quality control of data and algorithms; C. Morasso, F. Corsi, F. Sottotetti: data analysis and interpretation; S. Albasini: statistical analysis; C. Morasso, A. Caldarone, F. Piccotti: manuscript preparation; F. Sottotetti: manuscript editing; F. Corsi: manuscript review. 


\section{References:}

1. A. Gucalp, T.A. Traina, J.R. Eisner, J.S. Parker, S.R. Selitsky, B.H. Park, et al. Male breast cancer: a disease distinct from female breast cancer. Breast Cancer Research and Treatment 2019; 173:37-48.

2. M. Yalaza, A. İnan, M. Bozer. Male Breast Cancer. J Breast Health 2016; 12:1-8.

3. F. Wang, X. Shu, I. Meszoely, T. Pal, I.A. Mayer, Z. Yu, et al. Overall Mortality After Diagnosis of Breast Cancer in Men vs Women. JAMA Oncol 2019; 5:1589-1596.

4. S.H Giordano. Breast cancer in man. N Engl J Med 2018; 378:2311-2320.

5. S. Doyle, J. Steel, G. Porter. Imaging male breast cancer. Clinical Radiology 2011; 66:10791085.

6. H.J Butler, L. Ashton, B. Bird, G. Cinque, K. Curtis, J. Dorney, et al. Using Raman spectroscopy to characterize biological materials. Nature Protocols 2016; 11:664-687.

7. R. Vanna, C. Morasso, B. Marcinnò, F. Piccotti, E. Torti, D. Altamura, et al. Raman Spectroscopy Reveals That Biochemical Composition of Breast Microcalcifications Correlates with Histopathologic Features. Cancer Res 2020; 80:1762-1772.

8. A.S. Haka, K.E. Shafer-Peltier, M. Fitzmaurice, J. Crowe, R.R. Dasari, M.S. Feld. Identifying microcalcifications in benign and malignant breast lesions by probing differences in their chemical composition using Raman spectroscopy. Cancer Res 2002; 62:5375-5380.

9. R. Baker, K.D. Rogers, N. Shepherd, N. Stone. New relationships between breast microcalcifications and cancer. Br J Cancer 2010; 10:1034-9.

10. I. Barman, N.C. Dingari, A. Saha, S. McGee, L.H. Galindo, W. Liu, et al. Application of Raman spectroscopy to identify microcalcifications and underlying breast lesions at stereotactic core needle biopsy. Cancer Res 2013; 73:3206-15.

11. C. Morasso, D. Sproviero, M.C. Mimmi, M. Giannini, S. Gagliardi, R. Vanna, et al. Raman spectroscopy reveals biochemical differences in plasma derived extracellular vesicles from sporadic Amyotrophic Lateral Sclerosis patients. Nanomedicine 2020; 29:102249.

12. M. Scimeca, E. Giannini, C. Antonacci, C. A. Pistoiese, L. G. Spagnoli, E. Bonanno. Microcalcifications in breast cancer: an active phenomenon mediated by epithelial cells with mesenchymal characteristics. BMC Cancer 2014; 14:286.

13. J. Winston, I-T. Yeh, K. Evers, A.K. Friedman. Calcium Oxalate Is Associated With Benign Breast Tissue: Can We Avoid Biopsy? Am J of Clin Pathology 1993; 100:488-92.

14. M.J. Radi. Calcium oxalate crystals in breast biopsies. An overlooked form of microcalcification associated with benign breast disease. Arch Pathol Lab Med 1989; 113:1367-1369.

15. R.F. Cox, M.P. Morgan. Microcalcifications in breast cancer: Lessons from physiological mineralization. Bone 2013; 53:437-450. 


\section{Figures and Tables:}

Table 1 Number of male a) and female b) microcalcifications exhibiting at least one pixel of the mineral components described by the legend

a

HA WIT aCaCa Calcite $\mathrm{CaO}$

\begin{tabular}{l|ccccc}
\cline { 2 - 5 } HA & 40 & 2 & 0 & 2 & 1 \\
WCaCa & 2 & 0 & 0 & 1 \\
Calcite & & & 1 & 1 & 0 \\
CaO & & & & 3 & 0 \\
& & & & & 1
\end{tabular}

b HA WIT aCaCa Calcite CaO HA WIT $\mathrm{aCaCa}$

Calcite $\mathrm{CaO}$

$\begin{array}{ccccc}136 & 6 & 0 & 4 & 0 \\ & 6 & 0 & 0 & 0 \\ & & 0 & 0 & 0 \\ & & & 17 & 0 \\ & & & & 0\end{array}$
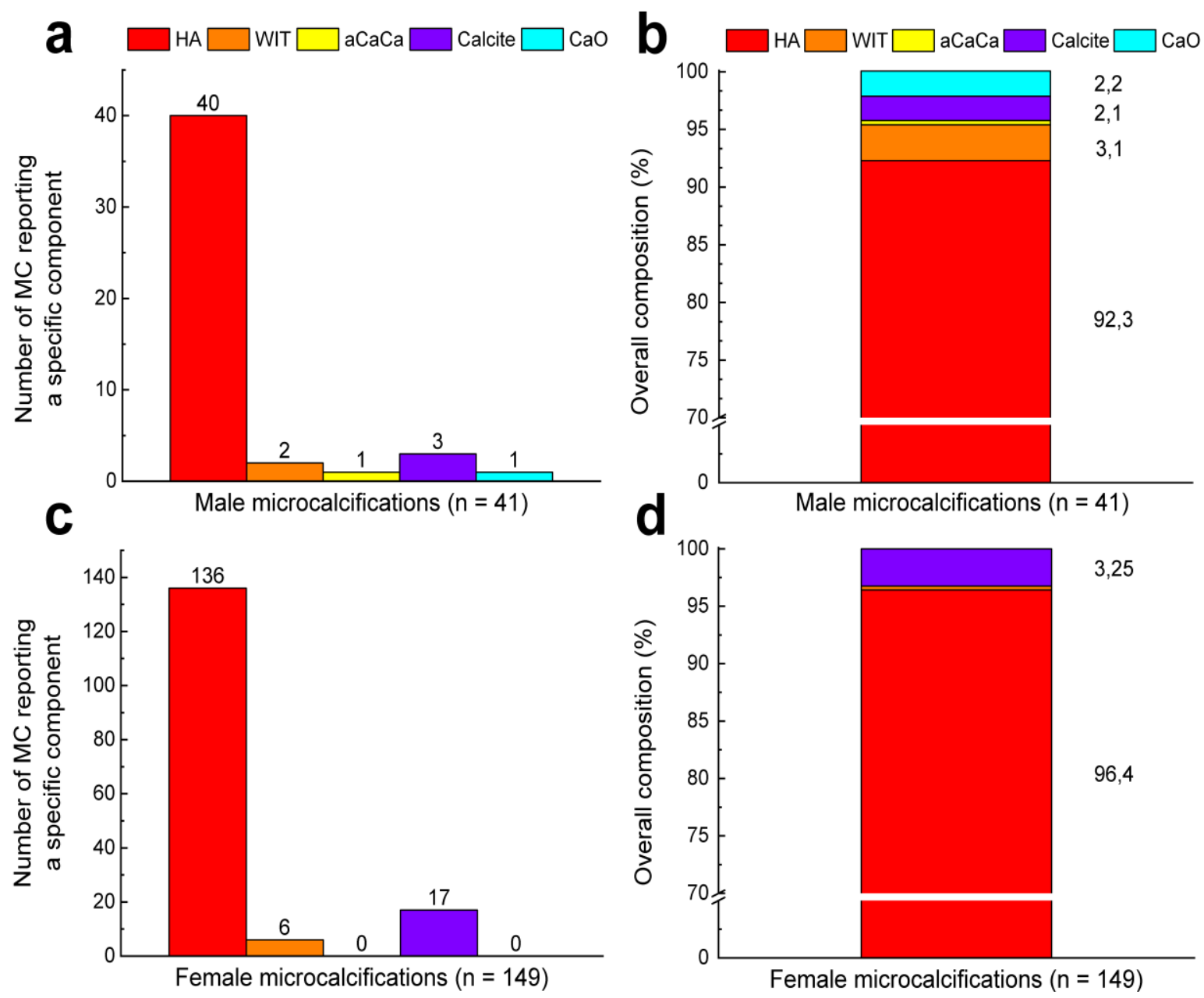

Fig. 1 Detailed composition of representative microcalcifications. Number of male a) and female c) microcalcifications exhibiting at least one pixel of the mineral components described by the legend. Overall composition of male b) and female d) microcalcifications, calculated, considering altogether all microcalcifications 

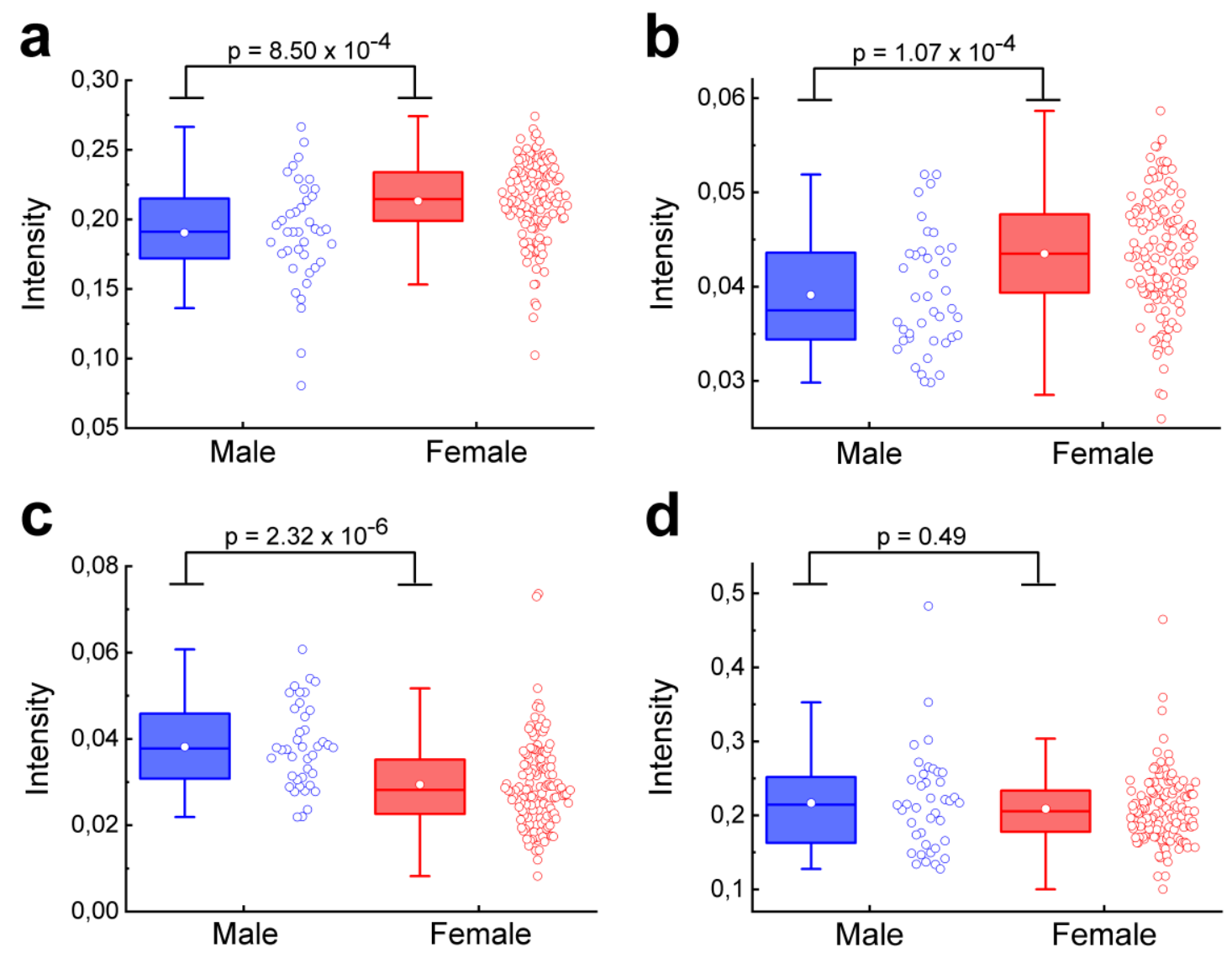

Fig. 2 a) Box plot reporting the intensity of the phosphate band $\left(960 \mathrm{~cm}^{-1}\right)$ in $\mathrm{MBC}$ and FBC. b) Box plot reporting the intensity of the carbonate band $\left(1075 \mathrm{~cm}^{-1}\right)$ in $\mathrm{MBC}$ and FBC. c) Box plot reporting the intensity of the band relative to the organic material $\left(1462 \mathrm{~cm}^{-1}\right)$ in $\mathrm{MBC}$ and $\mathrm{FBC}$. d) Ratio between the band of carbonate and phosphate in MBC and FBC 